\title{
Comparative Diagnosis of Serum IgG1 and Coproantigen ELISA for Fasciolosis Detection of Goats in Mexico
}

\author{
Abel Villa-Mancera, ${ }^{1}$ Pedro Molina-Mendoza, ${ }^{1}$ Karina Hernández-Guzmán, ${ }^{1}$ \\ Jaime Olivares-Pérez, ${ }^{2}$ Jorge Sarracent-Pérez, ${ }^{3}$ and José Zumaquero-Ríos ${ }^{4}$ \\ ${ }^{1}$ Laboratorio de Biotecnología Agropecuaria y Biología Molecular, Facultad de Medicina Veterinaria y Zootecnia, \\ Benemérita Universidad Autónoma de Puebla, 75482 Tecamachalco, PUE, Mexico \\ ${ }^{2}$ Unidad Académica de Medicina Veterinaria y Zootecnia, Universidad Autónoma de Guerrero, \\ 40660 Ciudad Altamirano, GRO, Mexico \\ ${ }^{3}$ Laboratorio de Hibridomas, Instituto de Medicina Tropical Pedro Kourí, La Habana, Cuba \\ ${ }^{4}$ Laboratorio de Parásitos y Vectores, Escuela de Biología, Benemérita Universidad Autónoma de Puebla, 72000 Puebla, PUE, Mexico
}

Correspondence should be addressed to Abel Villa-Mancera; abel.villa@gmail.com

Received 5 April 2016; Revised 27 June 2016; Accepted 10 July 2016

Academic Editor: David Piedrafita

Copyright (C) 2016 Abel Villa-Mancera et al. This is an open access article distributed under the Creative Commons Attribution License, which permits unrestricted use, distribution, and reproduction in any medium, provided the original work is properly cited.

\begin{abstract}
The objective of present study was to determine the prevalence of natural caprine fasciolosis in the Mixteca region of Mexico using coproantigen and serum IgG1 ELISA tests for comparative purposes. A total of 1070 serum and faecal samples were analyzed for IgG1 antibodies and coproantigens, using ELISA with E/S products as antigen and a monoclonal antibody-based sandwich ELISA. Prevalence of $73.46 \%$ was found using the serological ELISA and a percentage of 77.20 was found for coproantigen ELISA. The diagnostic sensitivity and specificity for serum ELISA were $86.7 \%$ and $96.4 \%$, and for the coproantigen ELISA they were $93.1 \%$ and $97.8 \%$, respectively. The seropositive samples were further categorized as low, medium, or high positivity. Results show a great proportion of low and medium positive goats when the serum ELISA test was used. Correlation coefficients between coproantigens and seropositivity were statistically significant $(P<0.01)$ for low seropositivity $(r=0.93)$ and medium seropositivity $(r=0.84)$. The accuracy of faecal antigen ELISA was higher compared to indirect ELISA serological test. Two ELISAs were shown to be useful for demonstrating the current status of $F$. hepatica infection in the endemic areas and can be employed in studies on epidemiology as well as anthelmintics treatment for preventing economic loss and the risk of transmission to humans.
\end{abstract}

\section{Introduction}

Fasciolosis is a foodborne zoonotic disease that affects grazing animals and humans. Fasciola hepatica causes global economic losses to the agriculture, estimated at over three billion US dollars every year [1]. At least 90 million people are at risk of infection and between 2.4 and 17 million individuals are currently infected [2]. The pathogenic trematode is widespread, causing infections in Bolivia, Peru, Ecuador, Egypt, and Iran [3-5].

Although caprine infection is lower than ovine or bovine fasciolosis, goats are extremely sensitive and susceptible to both natural and experimental infections [6, 7]. F. hepatica has been also detected in temperate cooler areas of high lands of tropical and subtropical regions [8]. Mexico possesses 8.6 million goats [9] located in areas with lowest human development index, with high potential to improve economy. High Fasciola prevalence in goats has been reported in the northwest Mexico using the indirect ELISA and sedimentation tests (43.0\% and $24.5 \%[10])$.

The economic importance of fasciolosis is attributed to the loss of livers in abattoirs [11], reduced feed efficiency and milk production, delayed animal growth, reproductive insufficiency, losses due to animal morbidity and mortality, and cost of treatment [12-15].

Rapid, early, and accurate diagnosis of the infection is key to studying the epidemiology of fascioliasis and the surveillance and control of this disease. Antemortem tools for 


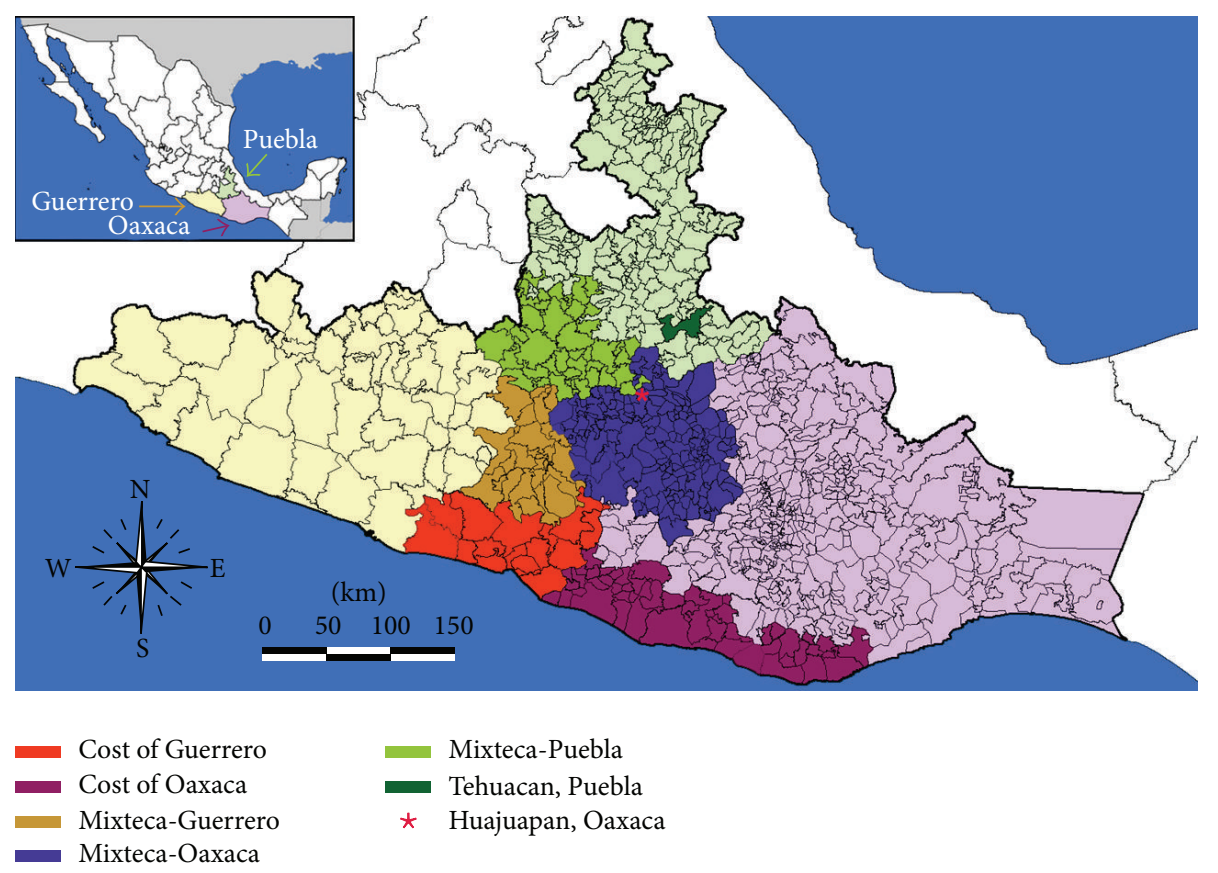

Figure 1: Map of the Mixteca region of Mexico and places mentioned in the text.

the detection of fasciolosis, ranging from copromicroscopic techniques to immunodiagnostics and molecular diagnostics, have been utilised $[16,17]$. Several studies had been performed on parasite antigens in faeces (coproantigens) and immunodiagnosis of $F$. hepatica infection in livestock (particularly sheep and cattle), while fewer studies had been done on diagnosis of liver fluke disease in goats [15, 17]. The aim of the present study was to compare the performances of monoclonal antibody-based sandwich ELISA in faeces (coproantigens) and serum IgG1 ELISA test for the diagnosis of Fasciola hepatica. This study was performed with a panel of samples from goats under the transhumant system in the Mixteca region of Mexico.

\section{Methods}

2.1. Study Area, Goat Breeds, and Sample Collection. The Mixteca region includes portions of three states of southern Mexico: (1) the western part of Oaxaca, (2) the eastern border of Guerrero, and (3) southern Puebla (Figure 1). It is divided into three subregions. The Mixteca Alta is located between the two other subregions; it is a high, temperate, and cool region of mountains and small valley. The Mixteca Baja refers to the arid regions at lower elevations of northern Mixteca Alta and extends north into Puebla. The Mixteca de la Costa covers the Pacific Coast to the northern mountains of Mixteca Alta region. The Mixteca region of Mexico has a great diversity of tree and shrub species which becomes important forage resources consumed by transhumance goats. Map of the Mixteca region of Mexico was generated using ArcGIS 10.1 (ESRI; Redlands, CA, USA).

The Creole goat is a dominant breed used for meat production in the Mixteca region. Other breeds such as
Boer, Nubia, Alpina, and their crosses have demonstrated considerable genetic merit to improve growth traits, mainly under extensive management conditions.

Producers purchase the goats grazing on the coast of Guerrero and Oaxaca and transported to grazing land in the Mixteca (May to November); then, the fattening goats were sent to Tehuacan, Puebla, and Huajuapan, Oaxaca, and sampled between October and November (autumn 2014). Blood and faecal samples from 1070 animals were collected from Mixteca-Guerrero and Puebla $(n=698)$ and MixtecaOaxaca $(n=372)$ and transported to the laboratory of Agricultural Biotechnology and Molecular Biology. Five grams of each faecal sample was processed individually using the sedimentation technique. Faecal eluates were prepared by adding $4 \mathrm{~mL}$ of phosphate-buffered saline/0.05\% (v/v) Tween 20 (PBS-T) to $1 \mathrm{~g}$ of fresh faeces in a centrifuge tube. The mixture was homogenized and then centrifuged at $900 \times \mathrm{g}$ for $5 \mathrm{~min}$ after which the supernatants were collected. Blood samples were centrifuged $(3,500 \times \mathrm{g})$ for $10 \mathrm{~min}$ and supernatant eluates and serum samples were stored at $-80^{\circ} \mathrm{C}$ until use.

Castrated male and female goats were fattened under an extensive production system, usually with grazing transhumance. Goats graze at high altitude during the rainy season and move to low altitude in cold weather, searching for better availability of fodder and weather conditions. Large herds graze on rented communal lands.

2.2. Adult F. hepatica Excretion/Secretion Products (E/S). Fasciola hepatica of caprine origin were acquired in our previous work [18]. Adult fluke E/S products were obtained by incubating mature parasites for $16 \mathrm{~h}$ at $37^{\circ} \mathrm{C}$ in RPMI-1640 supplemented with penicillin $(100 \mathrm{IU} / \mathrm{mL})$ and streptomycin 
$(100 \mu \mathrm{g} / \mathrm{mL})$. The supernatant was removed and centrifuged at $14000 \times \mathrm{g}$ for $30 \mathrm{~min}$ at $4^{\circ} \mathrm{C}$. E/S products were collected and concentrated in $10 \mathrm{kDa}$ cut-off membrane Amicon Ultra-15 centrifugal filter tubes (Millipore, USA) and stored in aliquots at $-80^{\circ} \mathrm{C}$.

\subsection{Positive and Negative Enzyme Linked Immunosorbent} Assay (ELISA) Controls. Four goats below 1 year of age receiving different doses of $F$. hepatica (250 and 300 metacercariae from infected Lymnaea cubensis host snail culture) acted as positive control. The presence of parasite eggs in faecal samples was performed using the sedimentation technique. Negative control was collected from parasite naïve goats (serum and faecal samples). All control serum samples were analyzed using an ELISA kit (DRG International Inc., USA) following the manufacturer's specifications to detect antibodies against $\mathrm{E} / \mathrm{S}$ products of $F$. hepatica.

2.4. Detection of Anti-F. hepatica IgG1 Antibodies in Serum by ELISA. The ELISA was optimized by checkerboard titration to determine the optimal concentration of antigen, serum, and conjugate dilutions. ELISA plates (Costar, Corning, NY, USA) were coated with $10 \mu \mathrm{g} / \mathrm{mL}$ of E/S products in $100 \mu \mathrm{L}$ of PBS and incubated overnight at $4^{\circ} \mathrm{C}$. After four washes with PBS-T, nonspecific binding sites were blocked with $200 \mu \mathrm{L}$ containing $1 \%$ bovine serum albumin (BSA) for $1 \mathrm{~h}$ at $37^{\circ} \mathrm{C}$. Negative and positive controls and serum samples were used at a dilution of $1: 400$ in $\mathrm{PBS}$ and incubated at $37^{\circ} \mathrm{C}$ for $1 \mathrm{~h}$. Microplates were washed four times with PBS-T and incubated with biotinylated sheep anti-bovine IgG1 isotype (1:10000, Abcam, USA; this antibody reacts with goats) for $1 \mathrm{~h}$ at room temperature in PBS-BSA 1\%. Plates were washed five times, each time with PBS-T. The detection of isotype IgG1 antibody was carried out using HRP-streptavidin conjugate (1:5000, Invitrogen, USA). Following incubation, plates were washed five times with PBS-T. Lastly, the reaction was developed by the addition of $100 \mu \mathrm{L}$ per well of TMB (Sigma, USA). The enzyme-substrate reaction was stopped with $50 \mu \mathrm{L}$ of $4 \mathrm{~N} \mathrm{H}_{2} \mathrm{SO}_{4}$. Absorbance was measured at $450 \mathrm{~nm}$ in ELISA reader (BioTek ELx800). All serum samples and negative and positive controls were tested in triplicate on each plate. The antibody levels were expressed as an optical density index (ODI) using the following formula: [(OD test sample)/(OD positive control) ] $\times 100$.

2.5. Detection of Coproantigen by Monoclonal Antibody(MoAb-) Based Sandwich ELISA. The optimal concentration of ES-78 MoAb [19] for coating plates for ELISA was determined by checkerboard titration using a positive-control and negative-control stool samples. For each step, $100 \mu \mathrm{L} /$ well was added unless otherwise stated. The plates were sensitized overnight at $4^{\circ} \mathrm{C}$ with MoAb $(10 \mu \mathrm{g} / \mathrm{mL}$ in PBS). After four washes with PBS-T, unbound sites were blocked with $200 \mu \mathrm{L}$ of $1 \% \mathrm{BSA}$ in PBS-T for $1 \mathrm{~h}$ at $37^{\circ} \mathrm{C}$. Undiluted faecal eluates were added and the plates were incubated at $37^{\circ} \mathrm{C}$ for $1 \mathrm{~h}$. After thorough washing as described above, HRP-conjugated rabbit anti-F. hepatica E/S products IgG (dilution 1:2500) in PBS-BSA $1 \%$ was added. The microplates were incubated for $1 \mathrm{~h}$ at $37^{\circ} \mathrm{C}$ and washed with PBS-T. Colour reaction was developed by the addition of TMB substrate (Sigma, USA) and read at $450 \mathrm{~nm}$.

2.6. Statistical Analysis. The diagnostic sensitivity, specificity, positive predictive values (PPV), and negative predictive values (NPV) were calculated [20]. Microscopy serves as the gold standard for true positive and true negative. Pearson correlation coefficients were used to assess the relationship among serum IgGl (low, medium, and high positivity) and E/S antigens in faeces. $P$ value greater than 0.05 was considered not significant and $P$ value less than 0.01 was considered highly significant. Statistical analysis was done using IBM SPSS 20 software package (SPSS Inc., Chicago, USA) for Windows.

\section{Results}

3.1. Sensitivity, Specificity, and Cut-Off Value of the ELISA. The optimum diagnostic sensitivity, specificity, and cut-off value were calculated using either sera or faeces tests (Table 1). The cut-off value was the 25 percent positivity (PP) for serum ELISA test; goats with a PP less than 25 were considered to be negative. Seropositivity was divided into 3 categories: low $(25 \leq \mathrm{PP} \leq 50)$, medium $(50 \leq \mathrm{PP} \leq 100)$, and high $(\mathrm{PP} \geq 100)$ positivity. For the coproantigen ELISA $\mathrm{OD}_{450}$ cut-off value for a positive result was defined at 0.5. Overall, the highest sensitivity and specificity were observed for the faecal antigen ELISA (93.1\% and 97.8\%, resp.), followed by the serological ELISA ( $86.7 \%$ and $96.4 \%$, resp.). Likewise, the diagnostic accuracy of the assay was $95.1 \%$ and $91.6 \%$. The serum and coproantigen ELISA showed high positive predictive values (proportion of goats that have fasciolosis with positive test results) of $98.5 \%$ and $99.3 \%$, with negative predictive values (proportion of goats that have fasciolosis with negative test results) of $52.6 \%$ and $51.2 \%$, respectively.

3.2. Prevalence of F. hepatica Infection. Coprological examination revealed prevalence of Fasciola hepatica in the Mixteca-Guerrero and Puebla of 59.45\% (415/698) and in Mixteca of Oaxaca of $49.19 \%$ (183/372) (Table 2). The overall prevalence of $F$. hepatica infection in the Mixteca region, measured by serum antibody and coproantigen, was $73.46 \%$ (786/1070) and $77.20 \%(826 / 1070)$, respectively. The prevalence detected by coproantigens analysis was consistently higher than that detected by the serological test. The prevalence of $F$. hepatica infection performed on serum samples by ELISA, categorized as low, medium, or high positive, respectively, was $30.00 \%, 41.12 \%$, and $2.34 \%$. The highest seroprevalence for medium positivity was detected in 440 of 1070 samples; whereas the lowest prevalence for high positivity was observed in 25 goats. The correlation coefficients between serum IgG1 prevalence and E/S antigens in faeces are shown in Table 3. Coproantigen ELISA was significantly correlated with low and medium positivity, though $r^{2}$ values were particularly high (Pearson's correlation coefficient $r=$ $0.93, r^{2}=0.86$, and $P<0.01$ and $r=0.84, r^{2}=0.70$, and $P<0.01$, resp.) but not significantly correlated with high 
TABLE 1: Evaluation of diagnostic values for detection of serum anti-F. hepatica IgG1 and E/S antigens in faeces.

\begin{tabular}{lcccc}
\hline & Sensitivity (\%) & Specificity (\%) & Positive predictive values (\%) & Negative predictive values (\%) \\
\hline Coproscopy & 75.2 & 98.3 & 98.2 & 56.5 \\
Serum ELISA & 86.7 & 96.4 & 98.5 & 52.6 \\
Coproantigen ELISA & 93.1 & 97.8 & 99.3 & 51.2 \\
\hline
\end{tabular}

TABLE 2: Prevalence of F. hepatica infection in goats from Mixteca region of Mexico by serum and coproantigen ELISA $(n=1070)$.

\begin{tabular}{lcc}
\hline & Number of goats samples & Prevalence $(\%)$ \\
\hline Coproscopy & & 59.45 \\
$\quad$ Mixteca-Guerrero and Puebla & 183 & 49.19 \\
$\quad$ Mixteca-Oaxaca & & 26.54 \\
Seroprevalence & 284 & 30.00 \\
$\quad$ Negative, $P P<25$ & 321 & 41.12 \\
Low positivity, $25 \leq \mathrm{PP} \leq 50$ & 440 & 2.34 \\
Medium positivity, $50 \leq \mathrm{PP} \leq 100$ & 25 & $73.46(49.59-52.21)$ \\
High positivity PP $\geq 100$ & 786 & $77.20(1.05-1.15)$ \\
$\quad$ Seropositivity $(95 \% \mathrm{CI})$ & 826 & \\
Coproantigen $(95 \%$ CI) & & \\
\hline
\end{tabular}

CI, confidence interval; PP, percent positivity.

TABLE 3: Pearson correlation coefficients between coproantigen and anti-F. hepatica IgG1 antibodies in goats (low, medium, and high positivity).

\begin{tabular}{lcccc}
\hline & Coproantigen & \multicolumn{3}{c}{ Seropositivity } \\
& & Low & Medium & High \\
\hline Coproantigen & - & & & \\
Low positivity & 0.93 & - & & \\
Medium positivity & 0.84 & 0.94 & - & \\
High positivity & -0.49 & -0.42 & -0.60 & - \\
\hline
\end{tabular}

positivity $(r=-0.49 ; P=0.32)$. Besides, the correlation among low positivity and medium positivity was higher and statistically significant $\left(r^{2}=0.89 ; P<0.01\right)$. A negative correlation was observed between high positivity and low positivity $(r=-0.42 ; P=0.40)$ and medium positivity $(r=-0.60 ; P=0.22)$.

\section{Discussion}

Traditional parasitological methods for diagnosis of Fasciola infections are usually based on the detection of parasite eggs in faeces, but trematode egg shedding is intermittent and irregular and does not detect immature stages of parasite. Copromicroscopy is labour-intensive and relatively unreliable. Detection of coproantigens by sandwich ELISA is a rapid, easy, and sensitive test compared to the liver fluke eggs in faeces [21]. Serological diagnosis by ELISA is the most sensitive and inexpensive technique for fascioliasis, detecting the infections earlier $[22,23]$.
In this study, a MoAb-based sandwich ELISA was employed for detection of circulating $F$. hepatica $\mathrm{E} / \mathrm{S}$ products (nonglycosylated antigen: 14, 24, 26, and $51 \mathrm{kDa}$ ) in stool samples and anti-F. hepatica IgG1 antibodies in serum by indirect ELISA. Natural infection with $F$. hepatica metacercariae has been shown to produce high levels of IgG1 antibodies and virtually no IgG2, thereby eliciting a nonprotective Th2 cell response [24]. Furthermore, the sensitivity and specificity of ES-78 MoAb-based ELISA in faeces were 93.1\% and 97.8\%, while in serum ELISA they were $86.7 \%$ and $96.4 \%$, respectively. Coproantigens were detected from 4 weeks after infection (wpi) in sheep using a MoAb-based sandwich ELISA [25]. E/S antigens in faeces of experimentally infected sheep could be detected from 4 wpi, with sensitivity of $93.3 \%$ [26]. Fluke E/S products recognized by antibodies in sera of infected goats can be detected from 2 wpi [27, 28]. The sensitivity and specificity values reported in the literature to detect anti-F. hepatica serum antibodies in sheep ranged from 68.2 to $100 \%$ and from 95 to $100 \%$, respectively $[29,30]$. In this trial, the diagnostic accuracy of ES-78 MoAb-based sandwich ELISA in stool was superior to serum samples (95.1\% versus 91.6\%); this could be due to the fact that coproantigens are less affected by immune complex formation [31].

The present study is the first report in the Mixteca region of Mexico in which indirect ELISA for the detection of serum antibodies was used to determine the prevalence of infection compared to a coproantigen test. The prevalence of infection was recorded to be higher for coproantigen ELISA (77.20\%) than indirect ELISA serological test (73.46\%). Additionally, $3.74 \%$ coproantigen-positive goats were found to be negative to serum IgG1 ELISA, which may indicate that the goat's immune system did not respond adequately to the antigenic 
stimulus from migrating or mature flukes [8]. Prevalence of $43.0 \%$ was detected using indirect ELISA in goats from a semidesert area in the northwest of Mexico [10]. In Galega goats from northwestern Spain, seroprevalence of $22.7 \%$ was found using capture ELISA (MM3 antigen comprised cathepsins L1 and L2 and a Kunitz-like protein) [32]. The prevalence of fasciolosis in Pakistan has been reported using a commercially available indirect ELISA kit (DRG, Germany) in goats and ranged between $4.08 \%$ and $49.36 \%$ [8, 33]. Over the past decade, the prevalence of $F$. hepatica infection has risen in part due to climate change, increased animal movement, and changing farming practices [34]. Other causes include age, sex, breed, husbandry, and protocols used for the treatment of fasciolosis [5]. The actual number of animal and human infections is likely to be much higher due to its asymptomatic nature, the limited availability of diagnostic tools, and the lack of systematic or coordinated reporting of infections, especially in undeveloped countries [35].

The anti-F. hepatica IgG1 ELISA result expressed as a positivity value (negative, low, medium, or high) estimates the concentration of antibodies in serum sample; it is a product of the prevalence and the intensity of infection in each infected goat. The results are in agreement with [36]. In the present study, the statistical analysis showed that the correlation between coproantigen and low seropositivity is very high (0.93). The highly significant positive correlation between coproantigens decreases as seropositivity increases. The presence of coproantigen indicates F. hepatica infection, while serology would indicate recent exposure. A significant correlation has been found among the presence of coproantigens, egg output and intensity of infection in sheep $[25,26]$, and cattle $[37,38]$.

Seasonally abundant parasitic infections have a profound influence on the migration patterns of transhumant groups (distance covered, number of movements, duration of stays in each location, and the direction of movement when the season changes), restricting their time in favourable grazing but unfavourable disease areas [39, 40]. The infection is present in areas with climatic and soil features for the establishment of the intermediate host snail. F. hepatica is transmitted mainly by snails of the Family Lymnaeidae which are frequent in the country [41].

The goats were bought from the coast of Guerrero and Oaxaca, mixed for a long grazing period, and may then acquire parasites from several farms. Livestock interact with wildlife on the grazing pasture and at drinking water points for the larger part of the year; fascioliosis affects a wide range of mammals [42]. Transhumance of goats has strongly and rapidly declined in our study area in the last few years and tended to prefer indoor confinement. Today, rural population is declining in Mexico due to migration to the United States and even those who remain in the goat meat sector are reluctant to practice transhumance.

\section{Conclusion}

These data showed that, with the use of ELISA based on E/S products as antigen and a monoclonal antibody, caprine fascioliasis can be diagnosed (sensitivity $\geq 86.7 \%$ and specificity $\geq 96.4 \%$ ) for arresting its negative impact on growth and productivity, preventing economic loss and the risk of transmission to humans. Further studies focusing on identifying the environmental factors and grazing management will be necessary for the establishment of an efficient control strategy of F. hepatica.

\section{Competing Interests}

The authors declare that they have no competing interests.

\section{Acknowledgments}

This study was supported by PRODEP (Programa para el Desarrollo Profesional Docente para el Tipo Superior) DSA 103.5/15/7449, Folio BUAP-PTC-410, DSA/103.5/15/10471, Folio BUAP-PTC-419, and Meritorious Autonomous University of Puebla (VIEP-VIMA-NAT-16-I).

\section{References}

[1] T. Spithill, P. M. Smooker, J. Sexton et al., "Development of vaccines against Fasciola hepatica," in Fasciolosis, pp. 377-410, CAB International, New York, NY, USA, 1999.

[2] J. Keiser and J. Utzinger, "Food-borne trematodiases," Clinical Microbiology Reviews, vol. 22, no. 3, pp. 466-483, 2009.

[3] M. B. Rokni, J. Massoud, S. M. O’Neill, M. Parkinson, and J. P. Dalton, "Diagnosis of human fasciolosis in the Gilan province of Northern Iran: application of cathepsin L-ELISA," Diagnostic Microbiology and Infectious Disease, vol. 44, no. 2, pp. 175-179, 2002.

[4] S. Mas-Coma, M. A. Valero, and M. D. Bargues, "Climate change effects on trematodiases, with emphasis on zoonotic fascioliasis and schistosomiasis," Veterinary Parasitology, vol. 163, no. 4, pp. 264-280, 2009.

[5] M. K. Khan, M. S. Sajid, H. Riaz et al., "The global burden of fasciolosis in domestic animals with an outlook on the contribution of new approaches for diagnosis and control," Parasitology Research, vol. 112, no. 7, pp. 2421-2430, 2013.

[6] J. J. Reddington, R. W. Leid, and R. B. Wescott, "The susceptibility of the goat to Fasciola hepatica infections," Veterinary Parasitology, vol. 19, no. 1-2, pp. 145-150, 1986.

[7] R. Zafra, L. Buffoni, R. A. Pérez-Écija et al., "Study of the local immune response to Fasciola hepatica in the liver and hepatic lymph nodes of goats immunised with a peptide of the Sm14 antigen," Research in Veterinary Science, vol. 87, no. 2, pp. 226232, 2009.

[8] K. Afshan, M. Qayyum, S. S. R. Rizvi, M. Mukhtar, M. Mushtaq, and J. E. Miller, "Serological and coprological comparison for rapid diagnosis of Fasciola hepatica infection in small ruminants from sub-tropical area of Pakistan," Small Ruminant Research, vol. 113, no. 1, pp. 267-272, 2013.

[9] SAGARPA-SIAP, Mexican System of Information about Agriculture and Fishing, Mexico City, Mexico, 2013, http://www.siap gob.mx/poblacion-ganadera/.

[10] J. A. Munguía-Xóchihua, F. Ibarra-Velarde, A. Ducoing-Watty, N. Montenegro-Cristino, and H. Quiroz-Romero, "Prevalence of Fasciola hepatica (ELISA and fecal analysis) in ruminants from a semi-desert area in the northwest of Mexico," Parasitology Research, vol. 101, no. 1, pp. 127-130, 2007. 
[11] H. Khoramian, M. Arbabi, M. M. Osqoi, M. Delavari, H. Hooshyar, and M. Asgari, "Prevalence of ruminants fascioliasis and their economic effects in Kashan, center of Iran," Asian Pacific Journal of Tropical Biomedicine, vol. 4, no. 11, pp. 918922, 2014.

[12] P. Torgerson and J. Claxton, Epidemiology and Control, Fasciolosis, 1999.

[13] S. Mas-Coma, M. D. Bargues, and M. A. Valero, "Fascioliasis and other plant-borne trematode zoonoses," International Journal for Parasitology, vol. 35, no. 11-12, pp. 1255-1278, 2005.

[14] D. Piedrafita, T. W. Spithill, R. E. Smith, and H. W. Raadsma, "Improving animal and human health through understanding liver fluke immunology," Parasite Immunology, vol. 32, no. 8, pp. 572-581, 2010.

[15] J. Charlier, J. Vercruysse, E. Morgan, J. Van Dijk, and D. J. L. Williams, "Recent advances in the diagnosis, impact on production and prediction of Fasciola hepatica in cattle," Parasitology, vol. 141, no. 3, pp. 326-335, 2014.

[16] F. A. Rojo-Vázquez, A. Meana, F. Valcárcel, and M. MartínezValladares, "Update on trematode infections in sheep," Veterinary Parasitology, vol. 189, no. 1, pp. 15-38, 2012.

[17] C. A. A. Rojas, A. R. Jex, R. B. Gasser, and J.-P. Y. Scheerlinck, "Techniques for the diagnosis of fasciola infections in animals: room for improvement," Advances in Parasitology, vol. 85, pp. 65-107, 2014.

[18] R. Marcet, A. Díaz, E. Arteaga, C. M. Finlay, and J. Sarracent, "Passive protection against fasciolosis in mice by immunization with a monoclonal antibody (ES-78 MoAb)," Parasite Immunology, vol. 24, no. 2, pp. 103-108, 2002.

[19] A. Villa-Mancera, A. Reynoso-Palomar, F. Utrera-Quintana, and L. Carreón-Luna, "Cathepsin L1 mimotopes with adjuvant Quil A induces a Th1/Th2 immune response and confers significant protection against Fasciola hepatica infection in goats," Parasitology Research, vol. 113, no. 1, pp. 243-250, 2014.

[20] R. H. Fletcher, S. W. Fletcher, and G. S. Fletcher, Clinical Epidemiology: The Essentials, Lippincott Williams \& Wilkins, Philadelphia, Pa, USA, 2012.

[21] N. E. Moustafa, M. H. Hegab, and M. M. Hassan, "Role of ELISA in early detection of Fasciola copro-antigens in experimentally infected animals," Journal of the Egyptian Society of Parasitology, vol. 28, no. 2, pp. 379-387, 1998.

[22] R. Sánchez-Andrade, A. Paz-Silva, J. Suárez, R. Panadero, P. Díez-Baños, and P. Morrondo, "Use of a sandwich-enzymelinked immunosorbent assay (SEA) for the diagnosis of natural Fasciola hepatica infection in cattle from Galicia (NW Spain)," Veterinary Parasitology, vol. 93, no. 1, pp. 39-46, 2000.

[23] M. P. Reichel, "Performance characteristics of an enzyme-linked immunosorbent assay for the detection of liver fluke (Fasciola hepatica) infection in sheep and cattle," Veterinary Parasitology, vol. 107, no. 1-2, pp. 65-72, 2002.

[24] J. P. Dalton, M. W. Robinson, G. Mulcahy, S. M. O’Neill, and S. Donnelly, "Immunomodulatory molecules of Fasciola hepatica: candidates for both vaccine and immunotherapeutic development," Veterinary Parasitology, vol. 195, no. 3-4, pp. 272285, 2013.

[25] B. E. Duménigo, A. M. Espino, C. M. Finlay, and M. Mezo, "Kinetics of antibody-based antigen detection in serum and faeces of sheep experimentally infected with Fasciola hepatica," Veterinary Parasitology, vol. 89, no. 1-2, pp. 153-161, 2000.

[26] C. Almazán, G. Avila, H. Quiroz, F. Ibarra, and P. Ochoa, "Effect of parasite burden on the detection of Fasciola hepatica antigens in sera and feces of experimentally infected sheep," Veterinary Parasitology, vol. 97, no. 2, pp. 101-112, 2001.

[27] A. Martínez, M. S. Martínez-Cruz, F. J. Martínez, P. N. Gutierrez, and S. Hernández, "Detection of antibodies to Fasciola hepatica excretory-secretory antigens in experimentally infected goats by enzyme immunosorbent assay," Veterinary Parasitology, vol. 62, no. 3-4, pp. 247-252, 1996.

[28] A. Martínez-Moreno, F. J. Martínez-Moreno, I. Acosta, P. N. Gutiérrez, C. Becerra, and S. Hernández, "Humoral and cellular immune responses to experimental Fasciola hepatica infections in goats," Parasitology Research, vol. 83, no. 7, pp. 680-686, 1997.

[29] C. R. Bautista-Garfias, M. E. López-Arellano, and A. SánchezAlbarrán, "A new method for serodiagnosis of sheep fascioliasis using helminth excretory-secretory products," Parasitology Research, vol. 76, no. 2, pp. 135-137, 1989.

[30] J. B. Cornelissen, W. A. de Leeuw, and P. J. van der Heijden, "Comparison of an indirect haemagglutination assay and an ELISA for diagnosing Fasciola hepatica in experimentally and naturally infected sheep," Veterinary Quarterly, vol. 14, no. 4, pp. 152-156, 1992.

[31] A. M. Espino, A. Díaz, A. Pérez, and C. M. Finlay, "Dynamics of antigenemia and coproantigens during a human Fasciola hepatica outbreak," Journal of Clinical Microbiology, vol. 36, no. 9, pp. 2723-2726, 1998.

[32] A. Pérez-Creo, P. Díaz, C. López et al., "Fasciola hepatica in goats from north-western Spain: risk factor analysis using a capture ELISA," The Veterinary Journal, vol. 208, pp. 104-105, 2016.

[33] R. Anjum, M. N. Khan, M. S. Sajid, and M. T. Javed, "Evaluation of commercial elisa kit for diagnosis of small ruminant Fascioliasis in Pakistan," Pakistan Journal of Agricultural Sciences, vol. 52, no. 1, pp. 183-189, 2015.

[34] J. Van Dijk, N. D. Sargison, F. Kenyon, and P. J. Skuce, “Climate change and infectious disease: helminthological challenges to farmed ruminants in temperate regions," Animal, vol. 4, no. 3, pp. 377-392, 2010.

[35] H. Toet, D. M. Piedrafita, and T. W. Spithill, "Liver fluke vaccines in ruminants: strategies, progress and future opportunities," International Journal for Parasitology, vol. 44, no. 12, pp. 915927, 2014.

[36] M. R. Salimi-Bejestani, R. Daniel, P. Cripps, S. Felstead, and D. J. L. Williams, "Evaluation of an enzyme-linked immunosorbent assay for detection of antibodies to Fasciola hepatica in milk," Veterinary Parasitology, vol. 149, no. 3-4, pp. 290-293, 2007.

[37] S. M. Abdel-Rahman, K. L. O’Reilly, and J. B. Malone, "Evaluation of a diagnostic monoclonal antibody-based capture enzyme-linked immunosorbent assay for detection of a 26to 28-kd Fasciola hepatica coproantigen in cattle," American Journal of Veterinary Research, vol. 59, no. 5, pp. 533-537, 1998.

[38] Y. M. Brockwell, T. W. Spithill, G. R. Anderson, V. Grillo, and N. C. Sangster, "Comparative kinetics of serological and coproantigen ELISA and faecal egg count in cattle experimentally infected with Fasciola hepatica and following treatment with triclabendazole," Veterinary Parasitology, vol. 196, no. 3-4, pp. 417-426, 2013.

[39] C. N. L. Macpherson, "The effect of transhumance on the epidemiology of animal diseases," Preventive Veterinary Medicine, vol. 25, no. 2, pp. 213-224, 1995.

[40] N. Xiao, S. Cai, M. Moritz, R. Garabed, L. W. Pomeroy, and Y. Deng, "Spatial and temporal characteristics of pastoral mobility in the far north region, cameroon: data analysis and modeling," PLoS ONE, vol. 10, no. 7, article e0131697, 2015. 
[41] I. Cruz-Mendoza, H. Quiroz-Romero, D. Correa, and G. Gómez-Espinoza, "Transmission dynamics of Fasciola hepatica in the Plateau Region of Mexico. Effect of weather and treatment of mammals under current farm management," Veterinary Parasitology, vol. 175, no. 1-2, pp. 73-79, 2011.

[42] L. Issia, S. Pietrokovsky, J. Sousa-Figueiredo, J. R. Stothard, and C. Wisnivesky-Colli, "Fasciola hepatica infections in livestock flock, guanacos and coypus in two wildlife reserves in Argentina," Veterinary Parasitology, vol. 165, no. 3-4, pp. 341344, 2009. 

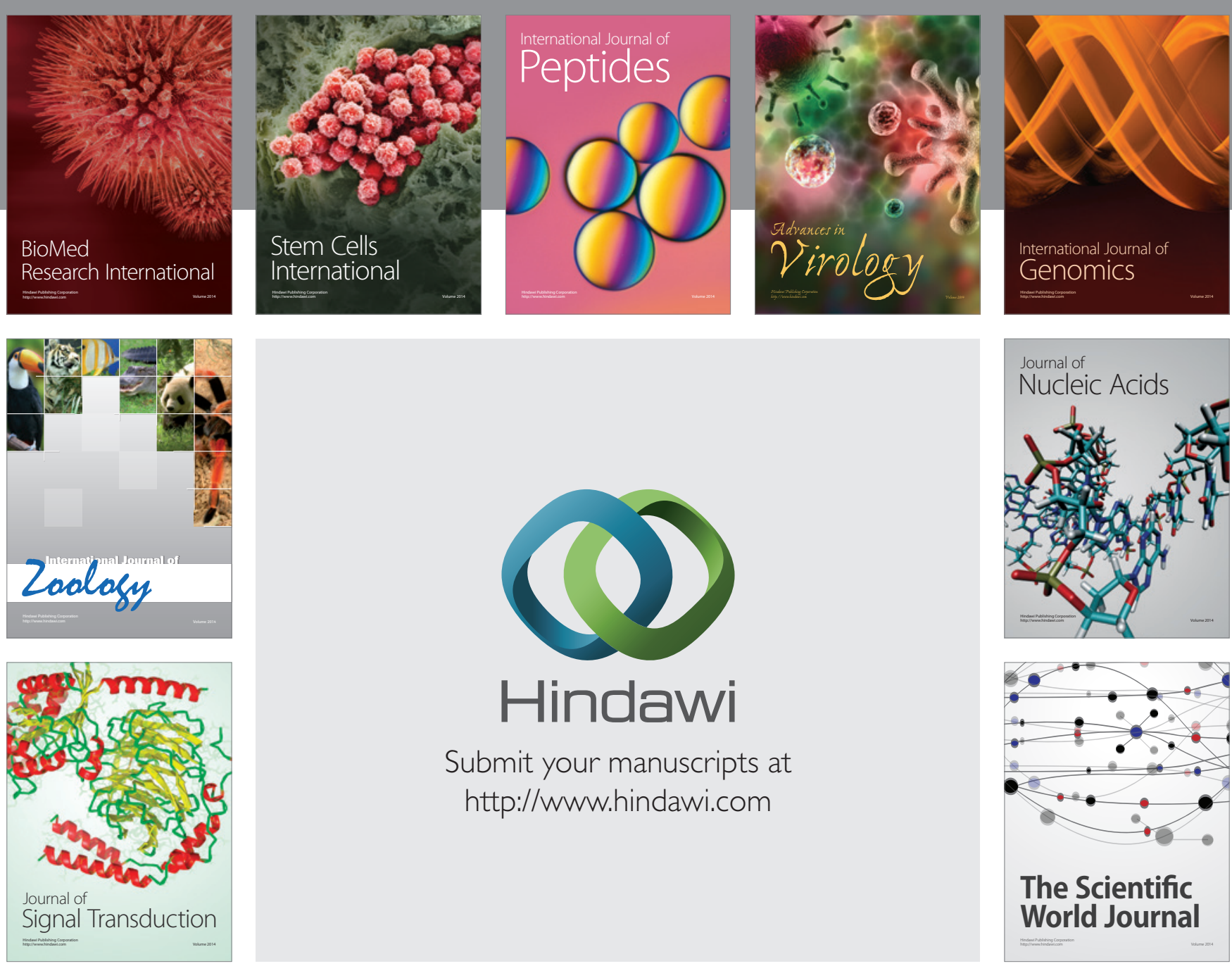

Submit your manuscripts at

http://www.hindawi.com
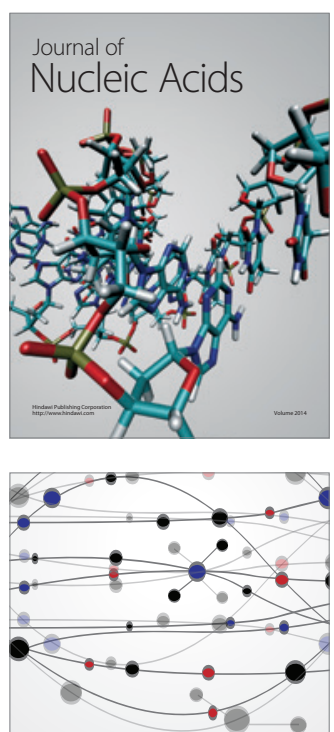

The Scientific World Journal
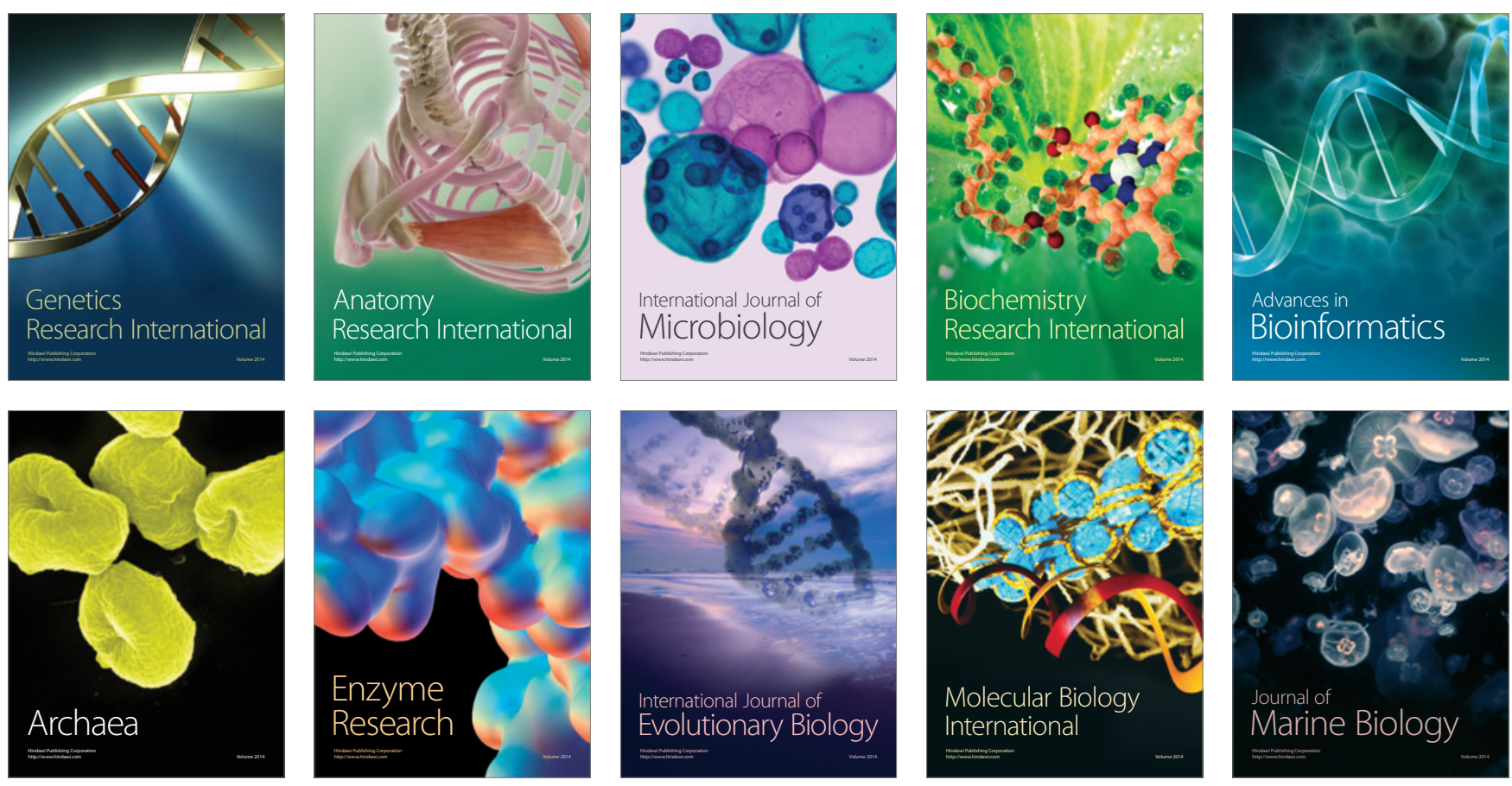